

\section{MODELO SIMPLIFICADO PARA EL CÁLCULO DE ILUMINANCIA POR LUZ NATURAL ÚTIL (UDI) EN ESPACIOS INDIVIDUALES PERIMETRALES DE CIELOS CLAROS. CASO DE ESTUDIO: MENDOZA, ARGENTINA.}

\section{A SIMPLIFIED MODEL FOR CALCULATING THE USEFUL DAYLIGHT ILLUMINANCE (UDI) IN INDIVIDUAL PERIMETER ROOMS UNDER CLEAR SKIES: A CASE STUDY IN MENDOZA, ARGENTINA.}

JUAN MANUEL MONTEOLIVA Instituto de Ambiente, Hábitat y Energía (INAHE) - Centro Científico Tecnológico Mendoza (CONICET) Mendoza, Argentina

jmonteoliva@mendoza-conicet.gob.ar

AYELÉN VILLALBA

Instituto de Ambiente Hábitat y Energía (INAHE) - Centro Científico Tecnológico Mendoza (CONICET) Mendoza, Argentina

avillalba@mendoza-conicet.gob.ar
ANDRÉS ACEÑA

Facultad de Ciencias Exactas y Naturales, Universidad Nacional de Cuyo - CONICET

Mendoza, Argentina

acena.andres@gmail.com

ANDREA PATTIN

Instituto de Ambiente, Hábitat y Energía (INAHE) - Centro Científico Tecnológico Mendoza (CONICET) Mendoza, Argentina

apattini@mendoza-conicet.gob.ar

\section{RESUMEN}

Una de las maneras más eficientes de reducir el consumo eléctrico por iluminación artificial, es a través del adecuado uso de la luz natural para la iluminación de espacios interiores. Sin embargo, las normativas vigentes que regulan el uso de la iluminación natural en espacios interiores se encuentran desactualizadas y en algunos casos no son sencillas de aplicar. Esto conduce a que los proyectistas no sean estimulados a buscar soluciones energéticamente eficientes que potencien el uso de la luz natural para iluminar espacios interiores durante las horas diurnas. El presente trabajo propone un modelo simplificado para el cálculo predictivo de la iluminancia por luz natural útil (UDI), para locales perimetrales individuales de cielos claros. Este modelo busca incorporar la precisión y validez de las simulaciones dinámicas en el estudio del comportamiento de la luz natural, a una herramienta de predicción sencilla y aplicable por profesionales en entornos reales.

Palabras clave

iluminación natural, diseño arquitectónico, legislación habitacional, ventanas, edificios de oficinas.

\section{ABSTRACT}

One of the most efficient ways to reduce the energy consumption of artificial lighting is through the proper use of daylight in indoor spaces. However, current regulations governing the use of daylight in indoor spaces are outdated and in some cases are not easy to apply. Thus, designers are not encouraged to seek energy efficient solutions that enhance the use of daylight to illuminate interior spaces during daytime hours. This paper proposes a simplified model for calculating the useful daylight illuminance (UDI) for individual perimeter rooms under clear skies. This model seeks to combine the precision and validity of dynamic simulations in the study of daylight, in a simple and appropriate prediction tool for professionals in real environments.

Keywords 


\section{INTRODUCCIÓN.}

A pesar de los avances alcanzados en el campo de la iluminación artificial, aún es difícil reducir el consumo de energía en los grandes edificios (Boubekri, 2006). Entre un 20\% y un 30\% del consumo eléctrico de los edificios no residenciales se destina a la iluminación artificial (Krarti, Erickson y Hillman, 2005). En Argentina, son efectivamente los sistemas de iluminación artificial los que generan el mayor consumo de energía en estas construcciones -no residenciales-. Le siguen los sistemas de refrigeración $y$, finalmente, los equipamientos de oficina -edificios comerciales y públicos- (Fundación Vida Silvestre Argentina, 2006). En este contexto, al cual se suman las crisis energéticas internacionales y nacionales, pasadas y actuales, se vuelve fundamental reflexionar nuevamente sobre esta problemática.

Una de las formas más eficientes de reducir el consumo eléctrico por iluminación artificial, es a través del adecuado uso de la luz natural para la iluminación de espacios interiores (Bodart et al., 2008). En ciudades donde predomina el cielo claro (Mendoza, Argentina; Kharga, Egipto; Tennant Creek, Australia; Phoenix, Yuma y Tucson, Estados Unidos; entre muchas otras ${ }^{1}$ ) y cuyo promedio anual es superior a las 2800 horas de sol, la radiación solar es un recurso sumamente útil en términos de eficacia luminosa²(Littlefair, 1985). En estas regiones la iluminación natural hace posible revertir parcialmente el consumo de energía eléctrica de los sistemas de iluminación artificial, y hasta, en algunos casos, prescindir de los mismos (Bodart y De Herde, 2002). Ello genera la necesidad de conocer con mayor precisión la cantidad de luz que ingresa a un edificio (Li et al., 2004) con el objetivo de diseñar estrategias de iluminación natural eficientes adaptadas a la región (Monteoliva, Villalba y Pattini, 2015).

La última década ha sido testigo de grandes avances producidos en la forma de evaluar y analizar el comportamiento de la iluminación natural en el interior de los espacios. Esta nueva perspectiva denominada paradigma dinámico (Ochoa, Aries y Hensen, 2012), incorpora el uso de métricas dinámicas (Reinhart, Mardaljevic y Rogers, 2006) y la caracterización de cielos a partir del uso de archivos climáticos y modelos de distribución de luminancia de cielo (Perez, Seals y Michalsky, 1993); dejando de lado indicadores y métodos basados en el paradigma estático o point-in-time (Ochoa, Aries y Hensen, 2012). Sin embargo, estos avances y el cambio producido en el sector de la construcción a través del uso de técnicas de diseño sustentable, no están reflejados en los marcos normativos vigentes. Esto conduce a que arquitectos, ingenieros y diseñadores de sistemas de iluminación híbridos -naturales y artificiales-, no sean estimulados a buscar soluciones eficientes (energéticamente) que potencien el uso de la luz natural para iluminar espacios interiores durante las horas diurnas (Monteoliva, 2014).

Existen dos aspectos que dificultan la aplicación de normas respecto al factor iluminación natural en códigos de eficiencia energética. En primer lugar, la iluminación natural es aún un recurso natural muy sub-explotado (Mardaljevic, 2006), por lo tanto, no es uno de los aspectos prioritarios en el diseño de edificios (Reinhart, 2002; Cantin y Dubois, 2011). En segundo lugar, toda evaluación, tanto pos-ocupacional como predictiva, requiere de una normativa o lineamiento de aplicación. No obstante, la compleja naturaleza de la fuente natural (sol) y su incidencia en el interior de los espacios dificultan alcanzar este objetivo.

Como consecuencia de la falta de regulaciones y en respuesta a las tendenciasarquitectónicas, en la actualidad los edificios en altura tienden a presentar fachadas vidriadas en su totalidad. Esta situación resulta particularmente crítica en aquellas regiones con cielos claros (CIE, 2001), donde la gran cantidad de radiación solar incidente requiere un control por demás intensivo. Diversas investigaciones sostienen que un elemento clave en el diseño de edificios no residenciales de bajo consumo energético, es la selección del área vidriada. Esto genera que dicha área sea una de las variables principales a considerar en la regulación de los códigos de eficiencia energética, la cual deberá mantener un equilibrio entre lo suficiente y lo no excesivo; de manera que se permita la visión al exterior, se evite el exceso de ganancia radiativa (solar y visible) y, como consecuencia, se gane en confort térmico y visual (Dubois, Blomsterberg y Flodberg, 2011).

La ausencia de normativas o lineamientos de aplicación genera en los organismos de normalización desafíos basados en la búsqueda e incorporación de nuevos indicadores de evaluación dinámica del factor iluminación natural, aplicables de acuerdo a las características climáticas de cada región (Monteoliva y Pattini, 2013). Asimismo, estudios recientes muestran muchas carencias en el modelado y losprocesos de simulación que hacen cuestionable por completo los procesos de diseño (Ibarra y Reinhart, 2009). Algunas de las principales insuficiencias limitaciones son: (i) interfaces gráficas

[1] Para mayor información visitar el sitio web Current results. Weather and sciences facts. (https://www.currentresults.com/WeatherExtremes/sunniest-places-countries-world.php) 


\begin{tabular}{|c|c|c|c|c|c|c|c|c|c|}
\hline WWR (\%) & 15 & 17.5 & 20 & 22.5 & 25 & 27.5 & 30 & 32.5 & 35 \\
\hline $\begin{array}{l}\text { tamaño } \\
\left(m^{2}\right)\end{array}$ & 1.8 & 2.1 & 2.4 & 2.7 & 3 & 3.3 & 3.6 & 3.9 & 4.2 \\
\hline $\begin{array}{l}\text { horizontal } \\
(H)\end{array}$ & & & & & & & & & \\
\hline ancho $(\mathrm{m})$ & 1.2 & 1.4 & 1.6 & 1.8 & 2 & 2.2 & 2.4 & 2.6 & 2.8 \\
\hline alto $(m)$ & 1.5 & 1.5 & 1.5 & 1.5 & 1.5 & 1.5 & 1.5 & 1.5 & 1.5 \\
\hline $\begin{array}{l}\text { vertical } \\
\text { (V) }\end{array}$ & & & & & & & & & \\
\hline ancho $(\mathrm{m})$ & 1.5 & 1.5 & 1.5 & 1.5 & 1.5 & 1.5 & 1.5 & 1.5 & 1.5 \\
\hline alto $(m)$ & 1.2 & 1.4 & 1.6 & 1.8 & 2 & 2.2 & 2.4 & 2.6 & 2.8 \\
\hline $\begin{array}{l}\text { escalar } \\
\text { (E) }\end{array}$ & & & & & & & & & \\
\hline ancho $(\mathrm{m})$ & 1.34 & 1.44 & 1.54 & 1.64 & 1.73 & 1.82 & 1.89 & 1.97 & 2.05 \\
\hline alto $(m)$ & 1.34 & 1.44 & 1.54 & 1.64 & 1.73 & 1.82 & 1.89 & 1.97 & 2.05 \\
\hline
\end{tabular}

Tabla 1. Descripción geométrica de las áreas vidriadas estudiadas. Fuente: Elaboración del autor.

no pensadas en el usuario ni en los tiempos requeridos de aprendizaje, (ii) tiempos de cálculos extremadamente extensos, sobre todo en técnicas avanzadas, como el trazado de rayos o raytracing, y (iii) procesos de simulación muycomplejos. En este escenario, surge la necesidad de disponer deindicadores que preferentemente no requieran de entornos de simulación para su predicción, y a la vez sean representativos del comportamiento dinámico de la luz natural. Esta nueva visión deberá contar necesariamente con la incorporación y compromiso de la ciencia y sus investigaciones.

En el marco de la problemática abordada, se propone aquíun modelo simplificado ${ }^{3}$ para el cálculo predictivo de la iluminancia por luz natural útil (UDI ${ }_{100-20001 x}$ ) (Nabil y Mardaljevic, 2005 y 2006), para locales perimetrales individuales bajo condiciones de cielo claro (Mendoza, Argentina), a partir del conocimiento del área vidriada, la disposición y la orientación de las ventanas. De este modo, se busca aportar al corpus teórico connuevas herramientas de evaluación dinámica de espacios iluminados por luz natural, sin la necesidad de recurrir a entornos de simulación.

\section{MÉTODO.}

\section{DISEÑO EXPERIMENTAL.}

A partir de un modelo virtual emplazado en la ciudad de Mendoza (Argentina) $\left(32^{\circ} 53^{\prime} 00^{\prime \prime} \mathrm{S}\right.$; 68 $\left.68^{\circ} 49^{\prime} 00^{\prime \prime} \mathrm{O}\right)$, se calcula la iluminancia por luz natural útil (UDI ${ }_{100-2000 \mid x}$ ) para 324 condiciones; resultado de la combinatoria de las siguientes variables: (i) disposición de la ventana: horizontal $(H)$, vertical (V) y escalar (E); (ii) área vidriada: $1.8 \mathrm{~m}^{2}\left(15 \% W W \mathrm{WR}^{4}\right), 2.1 \mathrm{~m}^{2}$ (17.5\% WWR), 2.4m² (20\% WWR), $2.7 \mathrm{~m}^{2}$ (22.5\% WWR), 3m² (25\% WWR), $3.3 \mathrm{~m}^{2}$ (27.5\% WWR), $3.6 \mathrm{~m}^{2}$ (30\% WWR), 3.9m² (32.5\% WWR)y $4.2 \mathrm{~m}^{2}$ (35\% WWR) (Tabla 1); (iii) orientaciones: $\mathrm{S} 0^{\circ}, 30^{\circ}, 60^{\circ}, 90^{\circ}, 120^{\circ}, 150^{\circ}, \mathrm{N} 180^{\circ}, 210^{\circ}, 240^{\circ}, 270^{\circ}, 300^{\circ}$, $330^{\circ}$, definidas en grados de acuerdo al azimut de la ventana.

[3] Este trabajo forma parte de un proyecto para el desarrollo de guías de diseño de edificios energéticamente eficientes en la ciudad de Mendoza, Argentina, a cargo de integrantes del Grupo de lluminación Natural del Instituto Nacional de Ambiente, Hábitat y Energía (INAHE) - Centro Científico Tecnológico Mendoza (CCT Mendoza).

[4] El rango de window to wall ratio (WWR) seleccionado se basa en diversos trabajos científicos y normas que recomiendan que el área vidriada de un espacio debe mantenerse en un rango de 15 a 40\% (Peter Lyons \& Associates, 2008; U. S. Department of Energy, 2014; Dubois y Flodberg, 2013) para que los consumos de energía no se eleven, debido a la excesiva ganancia solar. En los climas soleados, especialmente, se recomiendan valores cercanos al 15\%. Por supuesto que los valores recomendados varían de acuerdo a la orientación. 


\begin{tabular}{ccccc}
\hline & $\begin{array}{c}\text { Transmitancia } \\
\text { solar }\end{array}$ & $\begin{array}{c}\text { Reflectancia } \\
\text { solar }\end{array}$ & $\begin{array}{c}\text { Transmitancia } \\
\text { visible }\end{array}$ & $\begin{array}{c}\text { Reflectancia } \\
\text { visible }\end{array}$ \\
\hline vidrio simple claro & 0.83 & 0.07 & 0.89 & 0.08 \\
\hline
\end{tabular}

Tabla 2. Propiedades ópticas del vidrio seleccionado.

Fuente: Elaboración del autor.

\section{MÉTRICA DINÁMICA DE ILUMINACIÓN NATURAL ANALIZADA.}

La métricadinámicamétrica dinámica de iluminación natural considerada en este trabajo es la iluminancia por luz natural útil o useful daylight illuminance (UDI), métrica que surge de la modificación del indicador de luz natural autónoma o daylight autonomy (DA), concebida por Nabil y Mardaljevic (2005). La selección de este indicador permite identificar el porcentaje de ocurrencia de iluminancias dentro de un determinado rango. El UDI consta de tres rangos de iluminancias: <100 [lx], 100$2000[\mathrm{~lx}$ y y >2000 [lx], proporcionando créditos a los valores entre 100-2000 [lx], denominado "rango útil"; lo que sugiere que valores de iluminancia fuera de este rango "no son útiles". Para su medición se dispuso sobre el modelo virtual una grilla de 12 sensores a la altura del plano de trabajo $(h=0.80 \mathrm{~m})$, con una distribución de 3 por 4 nodos.

\section{SIMULACIONES DE ILUMINACIÓN NATURAL.}

Modelo virtual. El espacio modelado corresponde a una oficina típica $(6 \times 4 \mathrm{~m} ; \mathrm{h}=3 \mathrm{~m})$ con ventana unilateral centrada respecto del muro $(0,0)$. La ventana inicial cuenta con una superficie vidriada de $1.8 \mathrm{~m}^{2}$, la cual va aumentando de acuerdo a las tres disposiciones $\left(H, V\right.$ y $E$ ) en intervalos de $0.3 \mathrm{~m}^{2}$, alcanzando un área máxima de $4.2 \mathrm{~m}^{2}$. La caracterización fotométrica de los materiales del espacio interior de las salas, se realizó de acuerdo a los valores típicos de reflectancia difusa: pared $60 \%$, techo $80 \%$, piso $30 \%$. En la Tabla 2 se muestran los datos ópticos del vidrio seleccionado.

Parámetros de simulación. Las simulaciones de iluminación natural fueron desarrolladas en entorno RADIANCE (Ward y Shakespeare, 1998), integrado en la herramienta DIVA para Rhinoceros 3D (McNeel and Associates, 2010). DIVA es una herramienta de análisis predictivo dinámico del factor de iluminación natural y consumo energético (Jakubiec y Reinhart, 2012). Los parámetros de simulación empleados corresponden a una escena simple con elementos traslúcidos, trasparentes y opacos; y sin elementos complejos de iluminación natural (Mardaljevic, 2000): (ab) 5; (ad) 1000; (as) 100; (aa) 0.1; (ar) 300; (dt) 0 ; (ds) 0 .

Archivo de ocupación del espacio. Se generóen un archivo de ocupación del espacio (*.occ) de acuerdo al horario de uso más frecuente de las oficinas ubicadas en edificios no residenciales de la ciudad de Mendoza: lunes a viernes de 9:00 a 17:00.

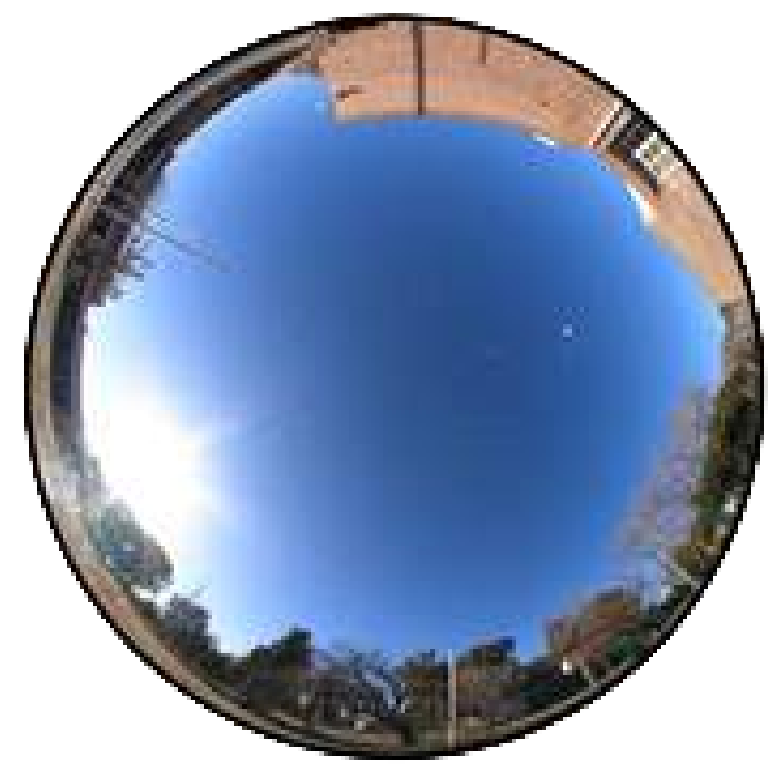

Figura 1. Imagen hemisférica del cielo de Mendoza, Argentina. Fuente: Fotografía del autor.

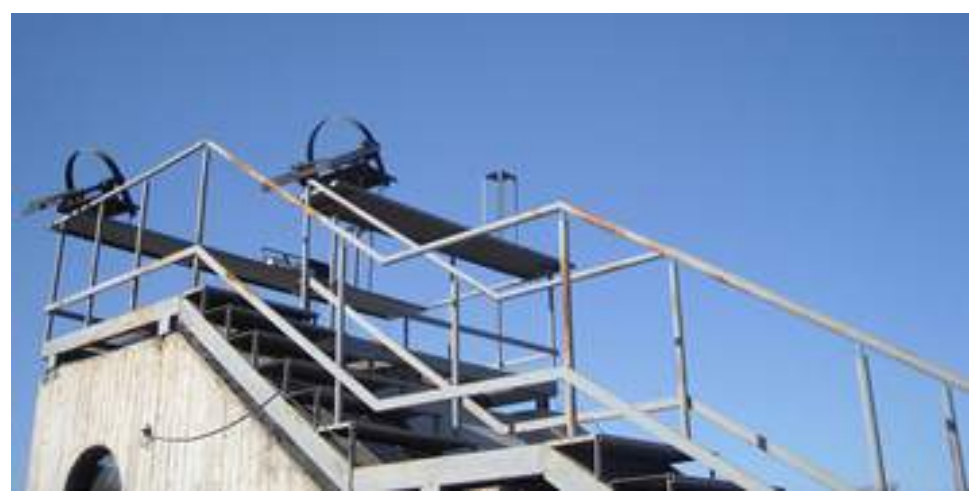

Figura 2. Estación de medición de iluminación natural del Instituto Nacional de Ambiente, Hábitat y Energía (INAHE), ubicado en el Centro Científico y Tecnológico Mendoza (CCT Mendoza). Fuente: Fotografía del autor. 
Archivos climáticos. Como se ha indicado, la ubicación seleccionada para el caso de estudio fue la ciudad de Mendoza (Argentina) (32 $\left.53^{\prime} 00^{\prime \prime} \mathrm{S} ; 68^{\circ} 49^{\prime} 00^{\prime \prime} \mathrm{O}\right)$. Esta ciudad se encuentra ubicada al oeste de la República Argentina en una región semi-árida, donde predominael cielo claro (Figura 1). Esta condición de cielo ofrece el 83\% del año días despejados o parcialmente despejados (Servicio Meteorológico Nacional Argentino para el período 1981-1990). Desde el punto de vista de la iluminación, Mendoza, como otras ciudades en el mundo, presenta valores de iluminancia global máximos medios de 90.000 lx en verano y de 30.000 lx invierno (Córica, 2009; Pattini 2009). El archivo de datos climáticos utilizado (ARG_MendozaCCT) corresponde a la ciudad de Mendoza (Argentina). Éste fue generado a partir de la información brindada por la estación de medición de Iluminación Natural del Instituto Nacional de Ambiente, Hábitat y Energía (INAHE), ubicado en el Centro Científico y Tecnológico Mendoza (CCT

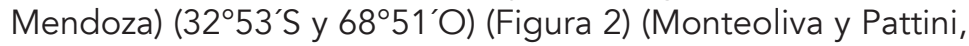
2013; Monteoliva, Villalba y Pattini, 2014).

\section{ANÁLISIS MATEMÁTICO.}

El procesamiento de los datos fue desarrollado en el entorno de SAGE Math(http://www.sagemath.org/). Éste es un software matemático de código abierto con licencia GPL producido desde la base de diferentes paquetes existentes: NumPy, SciPy, matplotlib y sympy, entre otros. Luego del análisis de los datos, fue posible generar ecuaciones cuadráticas de ajuste de los distintos valores obtenidos del indicador (UDI ${ }_{100-20001 x}$ ), con respecto al área vidriada en las diferentes orientaciones. Se calcularon, entonces, los errores asociados al ajuste, en particular, las desviaciones máximas entre el UDI simulado y el UDI ajustado. A partir de esos ajustes, y con la intención de completar el modelo, se llevó a cabo un ajuste de los coeficientes de las funciones cuadráticas como función de la orientación de la ventana. Dada la naturaleza angular de la orientación, se utilizó una aproximación de Fourier. Nuevamente, se analizaron los errores originados al utilizar la aproximación y, por último, se obtuvo una función predictiva del indicador UDI, gracias alconocimiento de la orientación y del área vidriada.

\section{RESULTADOS.}

En primera instancia, se analiza la dispersión de los valores de UDI, de acuerdo a las distintas disposiciones de la ventana, áreas y orientaciones (Figura 3). Como resultado de esta etapa, no se observan diferencias significativas entre las diversas disposiciones de la ventana $(\mathrm{H}, \mathrm{V}$ y $\mathrm{E})$ y los valores de UDI 20001 $x^{\prime}$ para una misma área vidriada y orientación de ventana. Debido a ello, se descarta del análisis la variable "disposición de la ventana". Asimismo, los valores obtenidos para las orientaciones este y oeste coinciden si el ángulo formado con el sur es el mismo, por lo tanto, el análisis se realiza en el rango de $0^{\circ}$ a $180^{\circ}$.

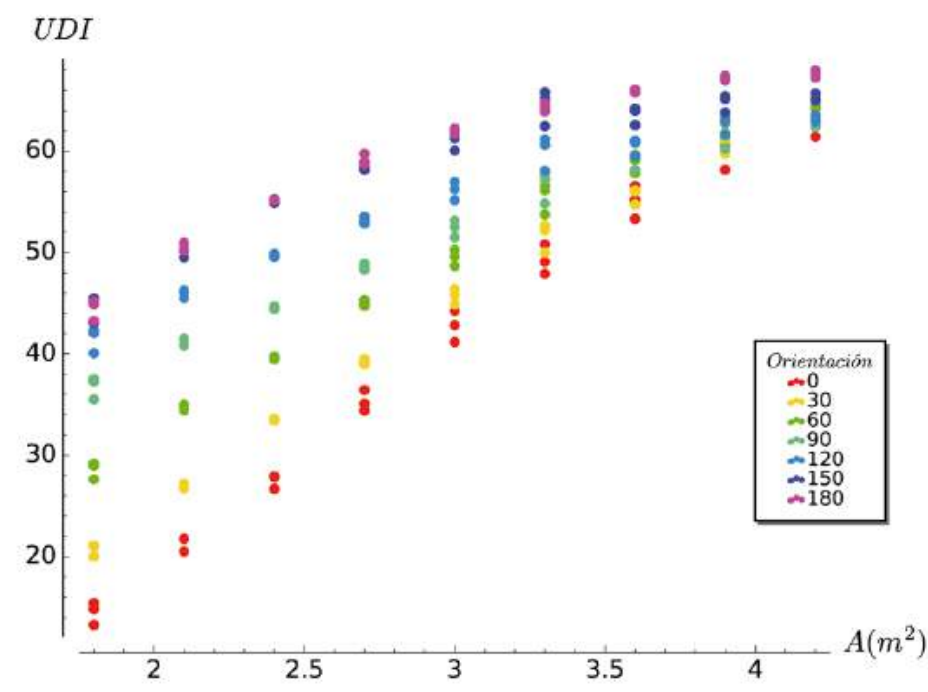

Figura 3. Gráfica de dispersión de los valores de UDI100-2000lx de acuerdo al área vidriada. Fuente: Elaboración del autor.

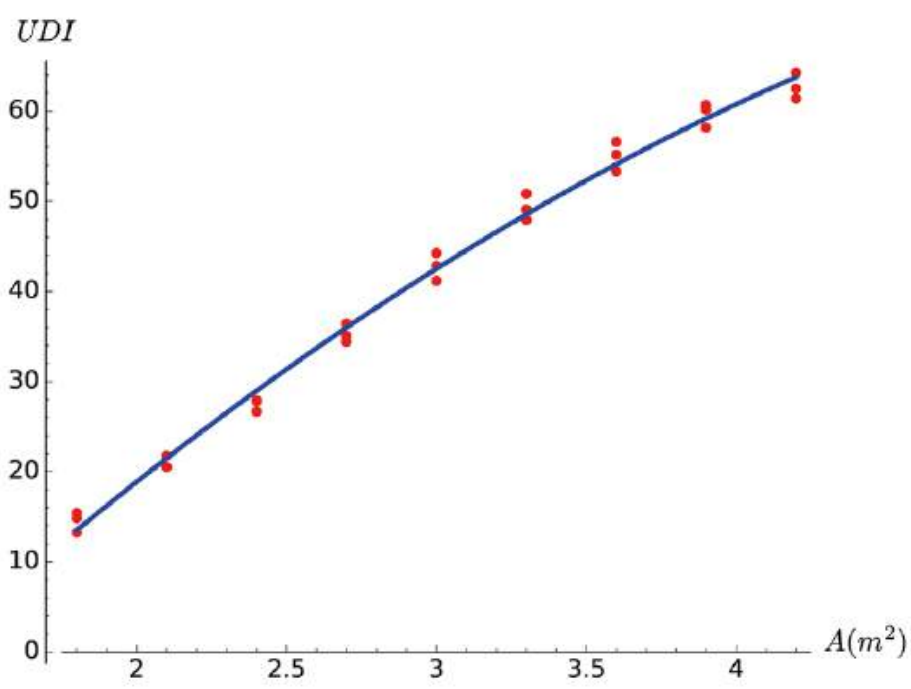

Figura 4. Datos y ajuste cuadrático para la orientación sur. Fuente: Elaboración del autor.

En principio, los datos por orientación están separados para analizar la dependencia entre el indicador UDI y el área vidriada. Se observa que en todos los casos el UDI aumenta al aumentar tambiénel área vidriada $(A)$, pero se trata de un incremento cada vez menor a medida que aumenta dicha área. Se concluye que un ajuste cuadrático resulta suficiente en todos los casos. Por consiguiente, una vezfijada la orientación, la expresión para el UDI queda definida de la siguiente forma:

$$
U D I(A)=a A^{2}+b A+c(1)
$$

Donde $a, b$ y c son los coeficientes del ajuste cuadrático.

En la Figura 4 se observan los datos y el ajuste cuadrático para la orientación sur. Los ajustes para las otras orientaciones son análogos. 


\begin{tabular}{ccccc}
\hline Orientación & a & b & c & $e$ \\
\hline $\mathrm{S} 0^{\circ}$ & -2.69 & 37.07 & 44.51 & $(e= \pm 2.4)$ \\
\hline $30^{\circ}-330^{\circ}$ & -2.53 & 33.39 & 31.71 & $(e= \pm 1.8)$ \\
\hline $60^{\circ}-300^{\circ}$ & -2.32 & 29.28 & 16.79 & $(e= \pm 2)$ \\
\hline $\mathrm{E}-\mathrm{O} 90^{\circ}-270^{\circ}$ & -1.97 & $22.99 *$ & -1.41 & $(e= \pm 2)$ \\
\hline $120^{\circ}-240^{\circ}$ & -2.84 & 26.21 & 3.36 & $(e= \pm 2.2)$ \\
\hline $50^{\circ}-210^{\circ}$ & -4.60 & 35.93 & 5.06 & $(e= \pm 2.4)$ \\
\hline $\mathrm{N} 180^{\circ}$ & -4.15 & 34.33 & 3.47 & $(e= \pm 1.6)$ \\
\hline
\end{tabular}

Tabla 3. Valores de a, b y c para las distintas orientaciones. Fuente: Elaboración del autor.

En la Tabla 3 se observan los coeficientes de los ajustes cuadráticos generados para los diferentes valores de UDI ${ }_{100}$ obtenidos para cada una de las orientaciones definidas $0^{\circ}, 30^{\circ}-330^{\circ}, 60^{\circ}-300^{\circ}, \mathrm{E}-\mathrm{O} 90^{\circ}-270^{\circ}, 120^{\circ}-240^{\circ}, 150^{\circ}-210^{\circ}$, $180^{\circ}$. Asimismo, se calculan los errores (e) cometidos con el ajuste, siendo estos las desviaciones máximas y mínimas entre los valores de UDI simulados y ajustados. Las curvas de los ajustes se presentan en la Figura 5. Como resultado de este procedimiento, es posible predecir el valor del indicador (UDI) de acuerdo al área vidriada para cada una de las orientaciones. Por ejemplo, tomando el caso de la orientación $\mathrm{N} 180^{\circ}$ y un área vidriada de $2 \mathrm{~m}^{2}$, el valor de UDI estimado es del $49 \%$ (línea naranja). Sin embargo, si la misma ventana estuviera orientada al sur $0^{\circ}$, para alcanzar un valor similar de UDI, el área vidriada debería ser de $3.3 \mathrm{~m}^{2}$ (línea azul) (Figura 5).

Si bien los ajustes encontrados representan un avance en el desarrollo del modelo matemático, todavía no se ha incorporado la dependencia angular del UDI en el mismo. Para esto, y dado que el ajuste cuadrático resulta satisfactorio para la dependencia con respecto al área, se busca ajustar los coeficientes $a, b$ y $c$. En el siguiente paso se va a considerar que los coeficientes $a, b$ y $c$ son funciones de la orientación $(\theta)$, de modo que la fórmula a emplear es la siguiente:

$$
\mathrm{UDI}(\mathrm{A}, \theta)=a(\theta) \mathrm{A}^{2}+\mathrm{b}(\theta) A+c(\theta)(2)
$$

Dada la naturaleza angular de la orientación, para realizar el ajuste de los coeficientes se utilizan funciones trigonométricas. De esta manera, se consideran los primeros términos, en lo que sería la expansión de Fourier de los coeficientes. Cabe señalar que también se tiene conocimiento deque la función es par con respecto a la orientación sur, por lo que sólo es necesario considerar los términos que contienen cosenos. Así, los coeficientes quedan expresados de la siguiente forma:

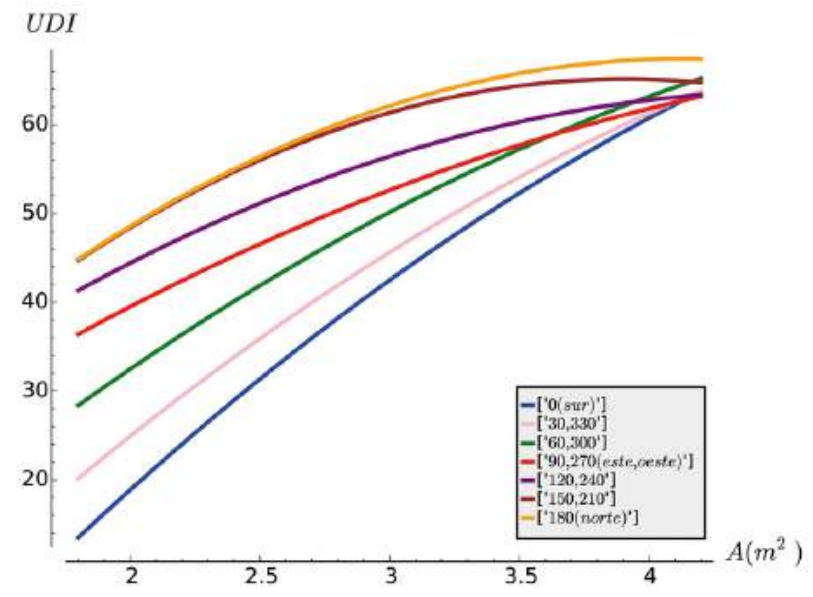

Figura 5. Curvas de ajustes obtenidos de UDI100-2000lx de acuerdo al área vidriada y la orientación. Fuente: Elaboración del autor.

$$
\begin{aligned}
& a(\theta)=a_{0}+a_{1} \cos (\theta)+a_{2} \cos (2 \theta)+a_{3} \cos (3 \theta), \text { (3) } \\
& b(\theta)=b_{0}+b_{1} \cos (\theta)+b_{2} \cos (2 \theta)+b_{3} \cos (3 \theta), \text { (4) } \\
& c(\theta)=c_{0}+c_{1} \cos (\theta)+c_{2} \cos (2 \theta)+c_{3} \cos (3 \theta) .
\end{aligned}
$$

En la Tabla 4 se detallan los valores encontrados para el ajuste y en las Figuras 6, 7 y 8 se muestran dichos ajustes. Con esto se completa el modelo. Al realizar el cálculo de errores para tal modelo, se obtienen errores máximos de \pm 3 . Se analiza, asimismo, la respuesta al utilizar ajustes con distinto número de términos para los coeficientes $a, b$ y $c$. Si se emplea un término más en la expansión de Fourier (5 términos), se obtienen errores de \pm 2.5 , que constituye el rango de errores en los ajustes sin la orientación. Con 3 términos, los errores son de \pm 4 , y con 2 términos, de \pm 5 . Finalmente, se resuelve usar el modelo con 4 términos, ya que permite un adecuado ajuste sin extender demasiado la cantidad de coeficientes empleados. 


\begin{tabular}{ccccc}
\hline & 0 & 1 & 2 & 3 \\
\hline$a$ & -2.91 & 0.87 & -0.74 & 0.02 \\
\hline$b$ & 30.42 & 0.57 & 6.22 & -0.22 \\
$c$ & -12.08 & -18.39 & -12.24 & -0.63 \\
\hline
\end{tabular}

Tabla 4.Valores encontrados para el ajuste de los coeficientes a, b y c. Fuente: Elaboración del autor.

3.1. Resumen del Modelo. A partir de los resultados obtenidos para conocer el UDI correspondiente a una cierta área vidriada (A) y cierta orientación ( $\theta)$, primero se utilizan las ecuaciones 3 , 4 y 5 para calcular los coeficientes a, b y c correspondientes a la orientación $\theta$, utilizando los coeficientes dados en la Tabla 4. Luego, se emplea la fórmula 2 para obtener el UDI de acuerdo al área vidriada. A continuación, se expone un ejemplo para el cálculo del UDI de un espacio orientado a $160^{\circ}$ con un área vidriada (A) de $3.2 \mathrm{~m}^{2}$.

$\mathrm{a}\left(160^{\circ}\right)=-2.91+0.87 \cos \left(160^{\circ}\right)-0.74 \cos \left(2 \times 160^{\circ}\right)+0.02 \cos \left(3 \times 160^{\circ}\right)=-4.30$

$b\left(160^{\circ}\right)=30.42+0.57 \cos \left(160^{\circ}\right)+6.22 \cos \left(2 \times 160^{\circ}\right)-0.22 \cos \left(3 \times 160^{\circ}\right)=34.76$

$c\left(160^{\circ}\right)=-12.08-18.39 \cos \left(160^{\circ}\right)-12.24 \cos \left(2 \times 160^{\circ}\right)-0.63 \cos \left(3 \times 160^{\circ}\right)=-3.86$

$\operatorname{UDI}\left(3.2,160^{\circ}\right)=-4.30 \times 3.2^{\wedge} 2+34.76 \times 3.2-3.86=63$

\section{DISCUSIÓN}

La naturaleza dinámica de la luz natural genera espacios lumínicamente dinámicos que no pueden ser caracterizados por un valor absoluto o instantáneo en el tiempo. El dinamismo propio de la luz natural, en términos de intensidad y temperatura de color (Monteoliva, Villalba y Pattini, 2014), dificulta su predicción y, con ello, su prescripción cuantitativa y cualitativa. Esto vuelve compleja la generación de un marco regulatorio para el factor 'iluminación natural' en espacios interiores. Si bien, como fue mencionado, esta problemática ha sido resuelta desde el campo de la simulación en entornos virtuales -paradigma dinámico-, aún no existen propuestas de indicadores para normativas regionales que no requieran de procesos de simulación y, de esa forma,faciliten el trabajo de profesionales proyectistas en campo.

El modelo generado es válido para espacios reducidos -no es aplicable en plantas abiertas- y en niveles superiores de los edificios o en bajas densidades urbanas. En escenarios de alta densidad urbana, las condiciones de iluminación se deben diferenciar en los niveles inferiores y superiores de los edificios (Córica, 2009; Villalba, 2014), en particular, en la

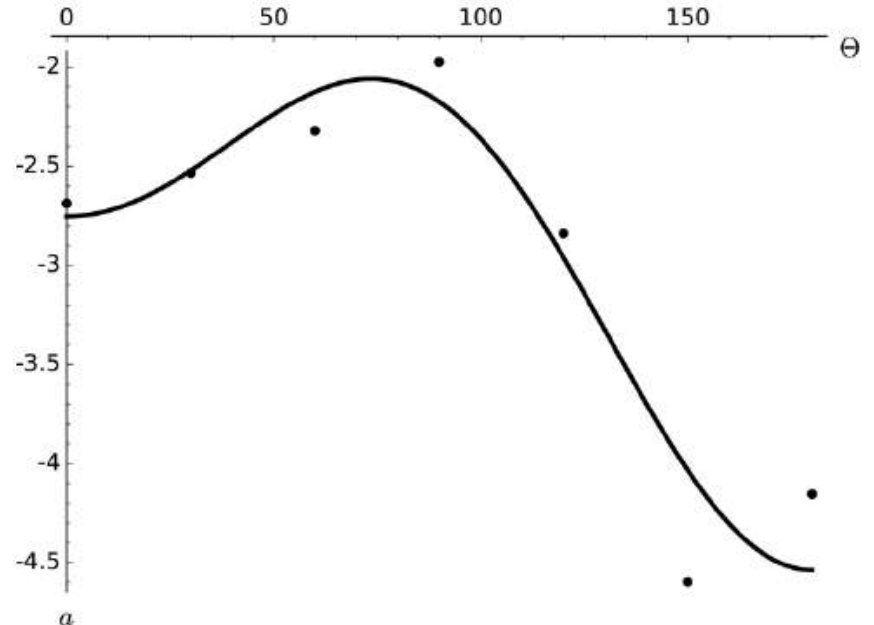

Figura 6. Curva de ajuste obtenida de los valores a para las distintas orientaciones. Fuente: Elaboración del autor.

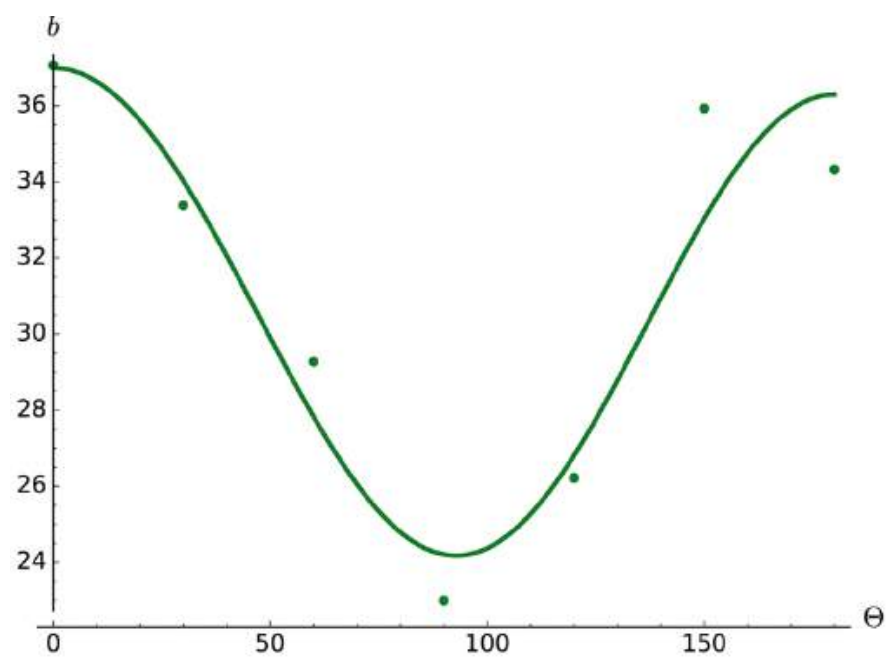

Figura 7. Curva de ajuste obtenida de los valores b para las distintas orientaciones. Fuente: Elaboración del autor.

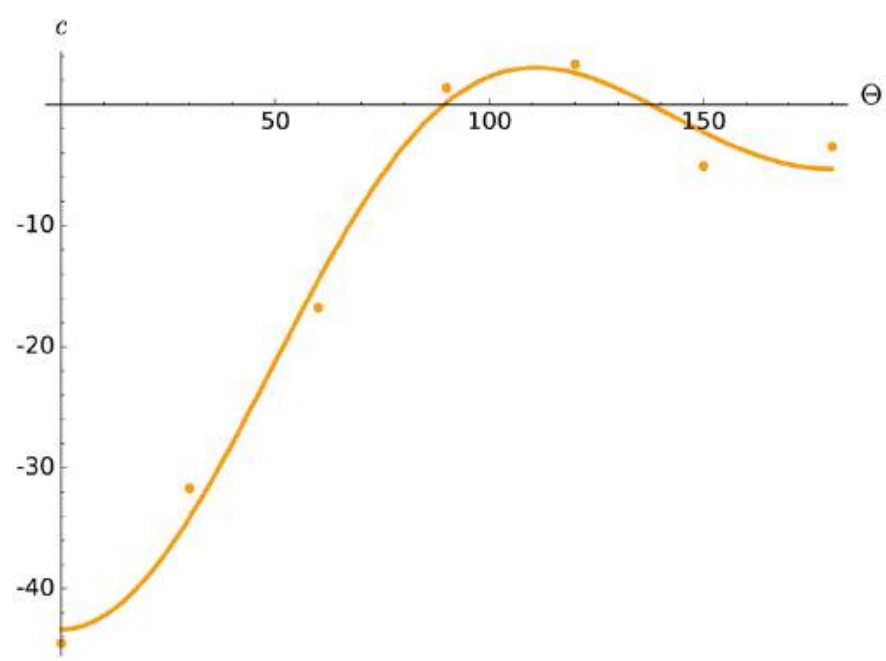

Figura 8. Curva de ajuste obtenida de los valores c para las distintas orientaciones. Fuente: Elaboración del autor. 
ciudades "oasis" (ciudad de Mendoza, Argentina, Figuras 9 y 10). En ellas, el árbol es el elemento de mitigación y de control ambiental estacional y el efecto de sombra es proyectado por estos túneles naturales consolidados por el bosque-urbano (Córica, 2009). Esto provoca que, durante el periodo estival, las fachadas norte, que se ubican por debajo de la copa de los forestales, no reciban radiación solar visible directa; mientras que las ubicadas por encima de la copa, sufran exposición solar directa. Asimismo, en periodos invernales, por la característica perenne de los forestales típicos de la región estudiada, la fachada norte se ve expuesta a sectores de luz difusa y a otros de luz directa, según el perfil de la fachada enfrentada y la altitud solar. Por otro lado, la fachada sur muestra niveles de iluminancia por debajo de los recomendados para ambas estaciones, ya que permanece en condición de sombra y no hay incidencia de radiación solar directa en los periodos de uso estudiados (Córica, 2009). Estas particularidades destacan la importancia de emplear un modelo sensible a las orientaciones y de diferenciar las recomendaciones de acuerdo al entorno urbano y a la disponibilidad de radiación solar de cada región. De igual modo, es importante considerar aspectos que tienen que ver con la conformación de los espacios interiores, tales como las oficinas de plantas abiertas y las reflectancias de las superficies interiores. A partir de las limitaciones mencionadas, se originan nuevos desafíos basados en la incorporación al modelo de nuevos indicadores y variables de análisis.

\section{CONCLUSIÓN}

El presente trabajo propone un modelo simplificado para el cálculo predictivo de la iluminancia por luz natural útil (UDI ${ }_{100-}$ 20001x), a partir del conocimiento del área y la orientación de la ventana, en locales perimetrales individuales de cielos claros, cuyos errores absolutos obtenidos son menores al $\pm 3 \%$. Este modelo busca integrarla precisión y validez de las simulaciones dinámicas en el estudio del comportamiento de la luz natural, a una herramienta de predicción sencilla y aplicable por profesionales en entornos reales. Es decir, se persigue ayudar a profesionales proyectistas sin dejar de lado la rigurosidad metodológica y potencial predictivo de los simuladores dinámicos.

Si bien resulta de interés incorporar nuevos parámetros al modelo propuesto, los resultados obtenidos son claramente alentadores hacia el objetivo de la sustentabilidad ambiental. Como es de conocimiento general, la relación existente entre la disponibilidad de la luz natural y el ahorro en los consumos energéticos es estrecha. Por este motivo, se aspira a contribuir a nuevas normativas regionales que promuevan el uso de la fuente natural para la iluminación de espacios interiores. De lograr este objetivo, se estará ayudando a concientizar sobre la aplicación y beneficios de espacios energéticamente eficientes, a través del adecuado uso de la iluminación natural.

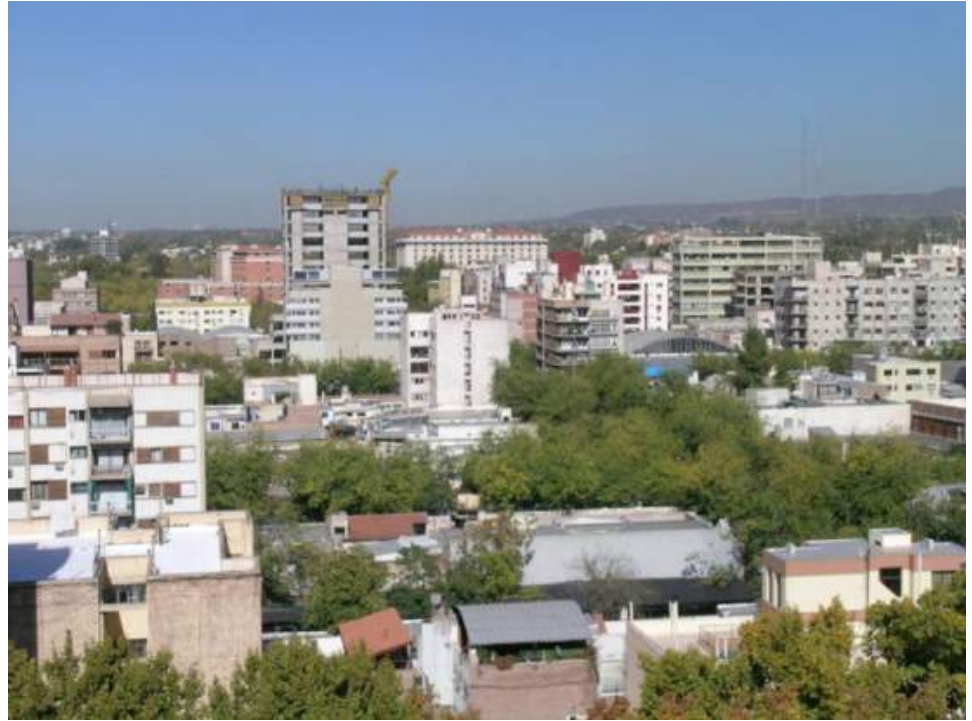

Figura 9. Ciudad "oasis" de Mendoza. Fuente: Fotografía del autor. Fuente: Figura realizada por el autor

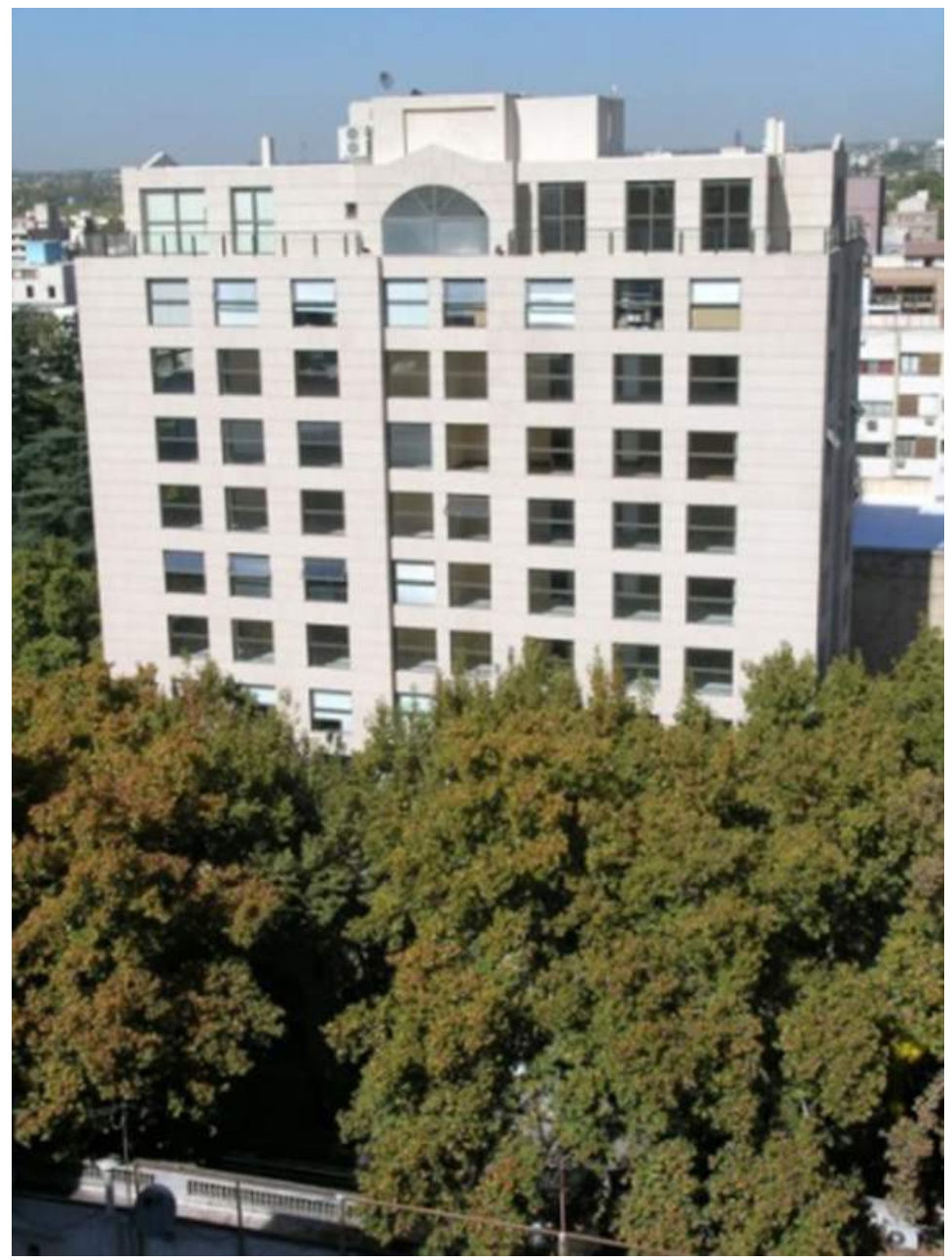

Figura 10. Diferentes condiciones de acceso a la luz natural por encima y por debajo de la copa de los forestales. Fuente: Fotografía del autor.

Fuente: Figura realizada por el autor 


\section{REFERENCIAS BIBLIOGRÁFICAS.}

BODART, Magali y DE HERDE, André. Global energy savings in office buildings by use of daylighting. Energy and Buildings, 2002, vol. 34, $n^{\circ} 5$, pp. 421-429.

BODART, Magali; DE PEÑARANDA, Rodrigue; DENEYER, Arnaud y FLAMANT, Gilles. Photometry and colorimetry characterisation of materials in daylighting evaluation tools. Building and Environment, 2008, vol. 43, n²12, pp. 2046-2058.

BOUBEKRI, Mohamed: An Overview of the Current State of Daylight Legislation. Human and Environment Systems, 2006, vol. 7, n 2, pp. 57-63

CANTIN, François y DUBOIS, Marie-Claude. Daylighting metrics based on illuminance, distribution, glare and directivity. Lighting Research and Technology, 2011, vol. 43, pp. 291-307.

CIE DS 011.1/E-2001. Spatial distribution of daylight - CIE standard general sky, Draft standard, CIE Central Bureau Vienna, 2001.

CÓRICA, Lorena. Comportamiento de la Luz Natural en Entornos Urbanos Representativos del Modelo Oasis en Regiones Áridas. Caso de estudio: Ciudad de Mendoza. Tesis Doctoral, Universidad Nacional de Tucumán, 2009.

DUBOIS, Marie-Claude; BLOMSTERBERG, Ake y FLODBERG, Kajsa. Towards zero energy office buildings in Northern Europe: Preliminary results of daylighting simulations. En: SB11 Helsinki World Sustainable Building Conference. Helsinki, Finland, 2011, pp. 18-21.

DUBOIS, Marie-Claude y FLODBERG, Kajsa. Daylight utilization in perimeter office rooms at high latitudes: Investigation by computer simulation. Lighting Research and Technology, 2013, vol. 45, issue 1, pp. 52-75.

Fundación Vida Silvestre Argentina (FVSA).Reducir emisiones ahorrando energía: escenarios energéticos para la Argentina (20062020) con políticas de eficiencia. Buenos Aires: Fundación Vida Silvestre Argentina, 2006.

IBARRA, Diego y REINHART, Christoph. Daylight factor simulations ¿How close do simulation beginners really get? En: Proceeding of the Eleven $^{\text {th }}$ International IBPSA Conference. Glasgow, Scotland, 2009, pp. 196-203.

JAKUBIEC, Alstan y REINHART, Christoph. The 'adaptive zone'. A concept for assessing glare throughout daylit spaces. Lighting Research and Technology, 2012, vol. 44, pp. 149-170.

KRARTI, Moncef; ERICKSON, Paul y HILLMAN, Timothy. A simplified method to estimate energy savings of artificial lighting use from daylighting. Building and Environment, 2005, vol. 40, n 6, pp. 747754.

LI, Danny; LAM, Joseph; LAU, Chris y HUAN T.W. Lighting and energy performance of solar film coating in air-conditioned cellular offices. Renewable Energy, 2004, vol. 29, nº 6, pp. 921-937.

LITTLEFAIR, Paul. The luminous efficacy of daylight: a review. Lighting Research and Technology, 1985, vol. 17, n² 4, pp. 162-182.

LYONS, Peter \& Associates. Building Energy Consultants Report to Australian Building Codes Board on Optimum Window Size for Energy Efficiency: BCA Volume One. Australia, 2008.
MARDALJEVIC, John. Daylight simulation: validation, sky models and daylight coefficients. Tesis Doctoral, Montfort University, 2000.

MARDALJEVIC, John. Examples of climate-based daylight modelling En: Proceedings of CIBSE National Conference 2006: Engineering the Future. London: Oval Cricket Ground, 2006, pp. 1-11.

MCNEEL, Robert and Associates. Rhinoceros Version 4.0.Service Release, 2010.

MONTEOLIVA, Juan. La dinámica de luz natural en cielos claros y su incidencia en el rendimiento atencional de los alumnos. Iluminación en aulas. Tesis Doctoral, Universidad Nacional de Tucumán, 2014.

MONTEOLIVA, Juan y PATTINI, Andrea. Iluminación natural en aulas. Análisis predictivo dinámico del rendimiento lumínico-energético en clima soleados. Ambiente construido 2013, vol. 13, n 4, pp. 235-248.

MONTEOLIVA, Juan;VILLALBA, Ayelén y PATTINI, Andrea. Uso de dispositivo de control solar en aulas: impacto en la simulación dinámica de la iluminación natural. Ambiente Construido, 2014, vol. $14, n^{\circ} 3$, pp. 43-58.

MONTEOLIVA, Juan; VILLALBA, Ayelén y PATTINI, Andrea. Estudio dinámico regional de la iluminación natural en espacios interiores. AREA, 2015, n²1, pp. 9-19.

NABIL, Azza y MARDALJEVIC, John. Useful daylight illuminance: A new paradigm to access daylight in buildings. Lighting Research and Technology, 2005, vol. 37, pp. 41-59.

NABIL, Azza y MARDALJEVIC, John. Useful daylight illuminances: A replacement for daylight factors. Energy and Buildings, 2006, vol. 38, pp. 905-913.

OCHOA, Carlos; ARIES, Myriam y HENSEN, Jan. State of the art in lighting simulation for building science: A literature review. Journal of Building Performance Simulation, 2012, vol. 5, n 4, pp. 209-233.

PATTINI, Andrea. La luz natural en las escuelas: aprovechamiento y control de la luz solar en aulas. Buenos Aires: Dunken, 2009.

PEREZ, Richard; SEALS, Robert y MICHALSKY, Joseph. All-weather model for sky luminance distribution- preliminary configuration and validation. Solar Energy, 1993, vol. 50, n³ pp. 235-245.

REINHART, Christoph. Effects of interior design on the daylight availability in open plan offices. En: Proceedings of the ACEEE Summer Study on Energy Efficient Buildings. Pacific Grove, CA, US, 2002, pp. 1-12.

REINHART, Christoph; MARDALJEVIC, John y ROGERS, Zack. Dynamic daylight performance metrics for sustainable building design. LEUKOS, 2006, vol. 3, n 1, pp. 7-31.

U.S. DEPARTMENT OF ENERGY. Advanced Energy Design Guides: Office Buildings.US, 2014.

VILLALBA, Ayelén. Control de luz solar directa en la envolvente edilicia en climas soleados. Su impacto en el consumo diurno de energía eléctrica para iluminación en edificios no residenciales de ciudad oasis. Tesis Doctoral: Universidad Nacional de Tucumán, 2014.

WARD, Greg y SHAKESPEARE, Rob. Rendering with Radiance: the art and science of lighting visualization. San Francisco: Morgan Kaufmann Publishers, 1998. 